\title{
Extracorporeal membrane oxygenation and surgical embolectomy for high-risk pulmonary embolism
}

\author{
To the Editor:
}

Patients with high-risk pulmonary embolism (PE) presenting with cardiogenic shock refractory to supportive measures have a high mortality $[1,2]$. Therapeutic success depends on rapid haemodynamic stabilisation and restoration of pulmonary blood flow. Thrombolytic therapy is the most widely used recanalisation strategy, but this treatment has substantial drawbacks including a high rate of bleeding complications and limited efficacy in patients with large embolic burden or in patients with recurrent $\mathrm{PE}$ presenting with acute-on-chronic events [3-8].

Surgical treatment of massive PE was introduced 110 years ago by Dr. Trendelenburg, but was widely abandoned due to high mortality rates $[4,9]$. The unsatisfactory surgical results were often related to the compromised clinical status of the patients, especially those who had already undergone thrombolysis and entered the operation room with advanced cardiogenic shock in need of cardiopulmonary resuscitation (CPR) [1, 2, 9-11]. Thus, the cornerstone for improving the results of surgical embolectomy may lie in the stabilisation of the preoperative haemodynamic condition, which can be achieved by veno-arterial extracorporeal membrane oxygenation (v-a ECMO) $[2,12,13]$.

At our institution, in November 2012, we introduced a pulmonary embolism response team (PERT) consisting of pneumologists, cardiologists, radiologists and cardiothoracic surgeons, and a standard operating procedure for patients with high-risk $\mathrm{PE}$.

Per protocol, thrombolytic therapy was not to be instituted prior to a PERT decision. v-a ECMO support was the preferred rescue therapy for patients who remained in cardiogenic shock or under CPR despite supportive measures.

Peripheral v-a ECMO was implanted via the femoral approach, preferably in awake, non-intubated patients to avoid further haemodynamic compromise due to anaesthesia and mechanical ventilation. A cannula for antegrade leg perfusion was inserted at the time of ECMO implant or as soon as possible after initial stabilisation. ECMO blood flow was usually kept at 2-3 L. $\mathrm{min}^{-1}$, paying particular attention to maintain pulmonary perfusion and arterial pulsatility. In patients with severely impaired upper body oxygenation, an additional inflow cannula was inserted in an internal jugular vein. The decision on definitive therapy after establishment of $\mathrm{v}$-a ECMO support was also made by the PERT with surgical treatment being the preferred strategy. The same strategy was used in patients retrieved from other hospitals by our mobile ECMO team.

During surgery, ECMO was switched to cardiopulmonary bypass $(\mathrm{CPB})$ using the previously implanted cannulas. An additional cannula was inserted in the superior vena cava for venous drainage. If the intraoperative findings showed acute-on-chronic PE, moderate hypothermic circulatory arrest was used to combine embolectomy with pulmonary endarterectomy (PEA). After completion of surgery, the cannula in the superior vena cava was removed and CPB was switched back to v-a ECMO support, which was extended postoperatively to prevent the development of lung reperfusion injury, and to allow recovery of cardiac function.

@ERSpublications

A treatment protocol for patients with high-risk pulmonary embolism, which avoids thrombolysis and establishes rapid haemodynamic stabilisation via $\mathrm{v}$-a ECMO support followed by semi-elective surgical embolectomy, is associated with excellent outcomes. http://ow.ly/rGNd30nBd22

Cite this article as: Ius F, Hoeper MM, Fegbeutel C, et al. Extracorporeal membrane oxygenation and surgical embolectomy for high-risk pulmonary embolism. Eur Respir J 2019; 53: 1801773 [https://doi.org/ 10.1183/13993003.01773-2018]. 
a)
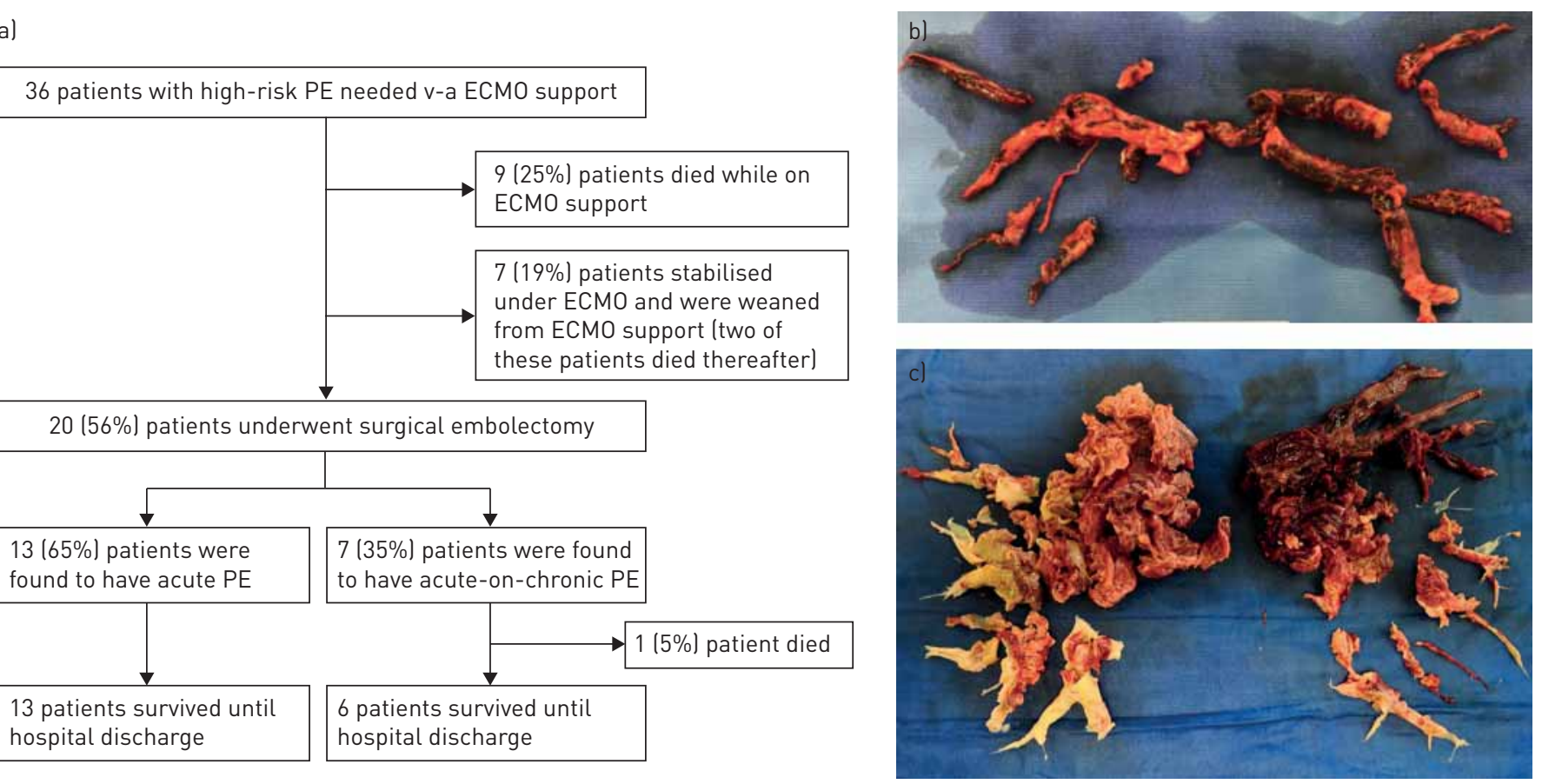

FIGURE 1 a) Flowchart for patient treatment disposition and b, c) surgically removed materials. Specimens showing b) fresh, red thrombus in a patient with acute pulmonary embolism (PE) and c) acute (red) and chronic (whitish) thrombus in a patient with acute-on-chronic PE. v-a ECMO: veno-arterial extracorporeal membrane oxygenation.

Here we present the results of pre- and perioperative ECMO therapy of 36 consecutive patients (36\% female; median age: 56 years, range 18-79 years) admitted for high-risk PE at our institution between November 2012 and August 2018. Follow-up ended on September 01, 2018. The treatment flowchart for these patients is shown in figure 1a.

In all patients, the initial computed tomography angiographies showed extensive central pulmonary emboli. Additional thrombotic material in the right heart chambers was visible in 8 (22\%) patients. 19 (53\%) patients had undergone unsuccessful thrombolysis or catheter-directed therapies, most of them in other hospitals prior to referral to our clinic. ECMO support was required because of persistent haemodynamic instability despite treatment with vasopressors and/or inotropes.

Nine (25\%) patients (CPR prior to ECMO, n=5) did not undergo surgery, because they did not stabilise $(n=7)$ or showed signs of severe hypoxic-ischaemic brain injury $(n=2)$. These patients all died.

Seven (19\%) other patients (CPR prior to ECMO, $n=2$ ) did not undergo surgery, because of severe obesity $(n=2)$, recent neurosurgery $(n=1)$, pregnancy $(n=1)$, unclear neurological status after CPR $(n=2)$, and lack of consent $(n=1)$. These patients stabilised on ECMO and anticoagulation alone $(n=6)$ or low-dose catheter-directed thrombolysis $(n=1)$, respectively, and were successfully weaned from ECMO after a median support time of 7 days. However, two patients died of sepsis prior to discharge from the hospital.

The remaining 20 (56\%) patients (CPR prior to ECMO, $\mathrm{n}=8$ ) underwent surgery. The median duration of $\mathrm{v}-\mathrm{a}$ ECMO support prior to surgery was 2 days (range: 0-9 days); two (10\%) patients required an additional venous cannula to treat hypoxaemia.

13 (65\%) patients underwent standard embolectomy (figure 1b), while seven (35\%) patients were intraoperatively found to have acute-on-chronic PE. These patients underwent combined embolectomy and PEA (figure 1c) and required moderate hypothermic circulatory arrest for a median of 28 min (range: 11-30 $\mathrm{min})$. Two (10\%) patients required coronary artery bypass grafting at the same time.

Postoperatively, all patients were transferred to the intensive care unit (ICU) under continued v-a ECMO support, which was weaned after a median of 5 days (range: 2-12 days). 12 (60\%) patients were extubated before ECMO weaning. Six (30\%) patients required prolonged mechanical ventilation and underwent tracheostomy. Three (15\%) patients required re-thoracotomy for bleeding and five $(25 \%)$ patients renal replacement therapy (temporary, $\mathrm{n}=4$ ). One $(5 \%)$, two $(10 \%)$ and three $(15 \%)$ patients showed lung reperfusion oedema, stroke and vascular complications after ECMO explantation (leg ischaemia, $\mathrm{n}=1$; femoral artery bleeding, $n=2$ ), respectively. Median ICU and hospital stay times were 11 days (range: 
6-34 days) and 22 days (range: 12-70 days), respectively. 17 (85\%) patients, including five who had required CPR prior to ECMO placement, had complete neurological recovery. One (5\%) patient died 17 days after surgery from septic multiorgan failure in hospital.

Taken together, the overall in-hospital survival rate in the present series was $67 \%$ (31\% in patients who did not undergo surgery and $95 \%$ in patients who underwent surgery, respectively). Among the 15 patients who had undergone CPR prior to ECMO placement, the overall survival rate was $60 \%$ (14\% in patients who did not undergo surgery and $100 \%$ in patients who underwent surgery). These results are comparable with recent case series on surgical treatment of patients with PE where rates of in-hospital mortality ranged from $3.6 \%$ to $31 \%[2,5-7,10-12,14]$ and neurological events from $2.9 \%$ to $20 \%[2,5,10-12,14]$.

Some authors have recently proposed that patients with high-risk PE could be treated by ECMO and anticoagulation alone (standalone ECMO) [15]. However, in a recent series published by MENEVEAU et al. [1], the mortality rate of patients treated with standalone ECMO was $78 \%$, while the mortality rate of patients treated with ECMO and surgical embolectomy was $29 \%$. Our results were comparable to the latter series with a $69 \%$ mortality rate in patients who received ECMO support but no surgical treatment. However, these results are affected by an inherent selection bias as the majority of the patients who were excluded from surgery were deemed to have a poor prognosis.

Of note, we identified previously unrecognised chronic thromboembolic changes in more than one third of patients undergoing surgery (figure 1c). This may explain, at least in part, the high proportion of patients who had failed thrombolytic therapy. These observations are of particular importance as they highlight a major limitation of thrombolytic therapy as well as the need to perform surgery in experienced centres, whenever possible. We acknowledge that surgical treatment for acute and acute-on-chronic PE is not available in most hospitals. However, ECMO support is becoming increasingly used and inter-hospital transfer of patients on ECMO has become feasible in many parts of the world.

In summary, our data show that a treatment protocol for patients with high-risk PE and refractory cardiogenic shock aiming at rapid haemodynamic stabilisation via v-a ECMO support followed by semi-elective surgical embolectomy may provide excellent outcomes.

Fabio Ius ${ }^{1,7}$, Marius M. Hoeper $\circledast^{2,3,4,7}$, Christine Fegbeutel ${ }^{1}$, Christian Kühn ${ }^{1,4}$, Karen Olsson ${ }^{2}$, Nurbol Koigeldiyev', Igor Tudorache ${ }^{1}$, Gregor Warnecke ${ }^{1,3,4}$, Jörg Optenhöfel ${ }^{1}$, Jakob Ono Puntigam ${ }^{1}$, Andreas Schäfer ${ }^{4,5}$, Bernhard C. Meyer ${ }^{3,6}$, Jan B. Hinrichs ${ }^{6}$, Johann Bauersachs ${ }^{4,5}$, Axel Haverich ${ }^{1,3,4}$ and Serghei Cebotari ${ }^{1,4}$

${ }^{1}$ Dept of Cardiothoracic, Transplant and Vascular Surgery, Hannover Medical School, Hannover, Germany. ${ }^{2}$ Dept of Respiratory Medicine, Hannover Medical School, Hannover, Germany. ${ }^{3}$ German Center for Lung Research (DZL), Hannover, Germany. ${ }^{4}$ Clinical Research Group (KFO 311), German Research Foundation, "Advanced cardiac and pulmonary failure: mechanical unloading and repair". ${ }^{5}$ Dept of Cardiology and Angiology, Cardiac Arrest Center and Advanced Heart Failure Unit, Hannover Medical School, Hannover, Germany. ${ }^{6}$ Dept of Diagnostic and Interventional Radiology, Hannover Medical School, Hannover, Germany. ${ }^{7}$ Both authors contributed equally.

Correspondence: Fabio Ius, Dept of Cardiothoracic, Transplant and Vascular Surgery, Hannover Medical School, Carl-Neuberg Strasse 1, 30625 Hannover, Germany. E-mail: ius.fabio@mh-hannover.de

Received: Sept 172018 | Accepted after revision: Jan 112019

Conflict of interest: F. Ius has nothing to disclose. M.M. Hoeper reports personal fees from Actelion, Bayer, GSK and Merck (lectures and consultations), outside the submitted work. C. Fegbeutel has nothing to disclose. C. Kühn has nothing to disclose. K. Olsson reports personal fees from Actelion (lectures), outside the submitted work. N. Koigeldiyev has nothing to disclose. I. Tudorache has nothing to disclose. G. Warnecke has nothing to disclose. J. Optenhöfel has nothing to disclose. J.O. Puntigam has nothing to disclose. A. Schäfer has nothing to disclose. B.C. Meyer has nothing to disclose. J.B. Hinrichs has nothing to disclose. J. Bauersachs has nothing to disclose. A. Haverich has nothing to disclose. S. Cebotari has nothing to disclose.

Support statement: M.M. Hoeper, C. Kühn, G. Warnecke, A. Schäfer, J. Bauersachs, A. Haverich, S. Cebotari are supported by the Deutsche Forschungsgemeinschaft, Clinical Research Group 311 (KFO 311) "Advanced cardiac and pulmonary failure: mechanical unloading and repair" (DFG; TP1). Funding information for this article has been deposited with the Crossref Funder Registry.

\section{References}

1 Meneveau N, Guillon B, Planquette B, et al. Outcomes after extracorporeal membrane oxygenation for the treatment of high-risk pulmonary embolism: a multicenter series of 52 cases. Eur Heart J 2018; 39: 4196-4204.

2 Al-Bawardy R, Rosenfield K, Borges J, et al. Extracorporeal membrane oxygenation in acute massive pulmonary embolism: a case series and review of the literature. Perfusion 2019; 34: 22-28.

3 Marshall PS, Tapson V, Jimenez D. Controversies in the management of life-threatening pulmonary embolism. Semin Respir Crit Care Med 2015; 36: 835-841.

4 Konstantinides SV, Torbicki A, Agnelli G, et al. 2014 ESC Guidelines on the diagnosis and management of acute pulmonary embolism. Eur Heart J 2014; 35: 3033-3080. 
5 Aymard T, Kadner A, Widmer A, et al. Massive pulmonary embolism: surgical emolectomy versus throbolytic therapy-should surgical indications be revisited? Eur J Cardiothorac Surg 2013; 43: 90-94.

6 Cho YH, Sung K, Kim WS, et al. Management of acute massive pulmonary embolism: is surgical embolectomy inferior to thrombolysis? Int J Cardiol 2016; 203: 579-583.

7 Lehnert P, Moller CH, Mortensen J, et al. Surgical embolectomy compared to thrombolysis in acute pulmonary embolism: morbidity and mortality. Eur J Cardiothorac Surg 2017; 51: 354-361.

8 Loyalka P, Ansari MZ, Cheema FH, et al. Surgical pulmonary embolectomy and catheter-based therapies for acute pulmonary embolism: a contemporary systematic review. J Thorac Cardiovasc Surg 2018; 156: 2155-2167.

9 Digonnet A, Moya-Plana A, Aubert S, et al. Acute pulmonary embolism: a current surgical approach. Interact Cardiovasc Surg 2007; 6: 27-29.

10 Neely RC, Byrne JG, Gosev I, et al. Surgical embolectomy for acute massive and submassive pulmonary embolism in a series of 115 patients. Ann Thorac Surg 2015; 100: 1245-1252.

11 Keeling WB, Sundt T, Leacche M, et al. Outcomes after surgical pulmonary embolectomy for acute pulmonary embolus: a multi-institutional study. Ann Thorac Surg 2016; 102: 1498-1502.

$12 \mathrm{Wu}$ MY, Liu YC, Tseng YH, et al. Pulmonary embolectomy in high-risk acute pulmonary embolism: the effectiveness of a comprehensive therapeutic algorithm including extracorporeal life support. Resuscitation 2013; 84: 1365-1370.

13 Weinberg A, Tapson VF, Ramzy D. Massive pulmonary embolism: extracorporeal membrane oxygenation and surgical pulmonary embolectomy. Semin Respir Crit Care Med 2017; 38: 66-72.

14 Vohra HA, Whistance RN, Mattam K, et al. Early and late clinical outcomes of pulmonary embolectomy for acute massive pulmonary embolism. Ann Thorac Surg 2010; 90: 1747-1752.

15 Corsi F, Lebreton G, Brechot N, et al. Life-threatening massive pulmonary embolism rescued by venoarterialextracorporeal membrane oxygenation. Crit Care 2017; 21: 76. 